Title: Benign Prostatic Hyperplasia (BPH)

Author: Travis Dunn, MS3

Date: December 2, 2009

Key words: Benign Prostatic Hyperplasia, Benign Prostatic Hypertrophy, Prostate Enlargement, BPH

Abstract: Saw palmetto is no more effective at relieving the symptoms of BPH (as measured on the American Urologic Association Symptom Inventory) than placebo. It is also not more effective than placebo at increasing peak urinary flow rates or decreasing prostate size.

This document was created by a medical student enrolled in the Primary Care Clerkship at the University of Minnesota Medical School as part of the course project. The aim of the project is to present information on a medical topic in the format of a patient education handout. It does not necessarily reflect the views of the University of Minnesota Medical School physicians and faculty. These materials are provided for informational purposes only and are in no way intended to take the place of the advice and recommendations of your personal health care provider. The information provided may no longer be up to date since it has not been reviewed since the date of creation. The information provided should not be used to diagnose a health problem or disease, or as a means of determining treatment. In the event of a medical emergency, immediately contact a doctor or call 911. 
For More Information on

Benign Prostatic Hyperplasia:

Mayo Clinic Website www.mayoclinic.org/bph

Family Doctor Website

http://familydoctor.org/online/famdocen

/home/men/prostate/148.html

Developed by

Travis Dunn, MS3

\section{Benign Prostatic Hyperplasia (BPH)}

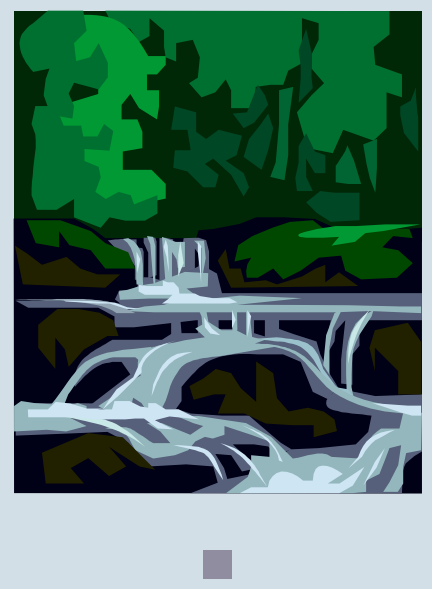

University of Minnesota Primary Care Clerkship Patient Education Tool 


\section{What is $B P H$ ?}

Benign prostatic hyperplasia (also known as BPH) is a condition that affects the prostate gland in men, causing the gland to grow in size. BPH is a fairly common condition, especially in men middle aged and older. The older a man gets, the more likely he is to develop BPH.

"Hyperplasia" refers to the enlargement of the prostate, and "benign" means that the enlargement is not caused by cancer or infection.

The urethra (the tube that drains urine from the bladder) passes through the prostate gland. As the prostate grows in size, it can begin to block the flow of urine through the urethra.

What are the symptoms of $B P H$ ? Symptoms of BPH may begin gradually, and include:

- Urinating more frequently than normal

- Having to urinate during the night

- Suddenly feeling the need to urinate

- Weak urine stream

- Urine stream that stops and starts

- Straining to pass urine 